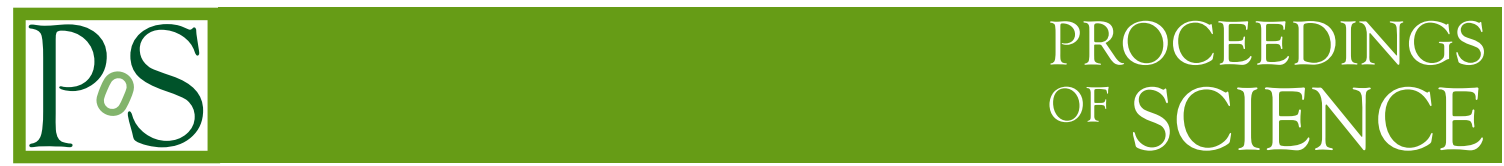

\title{
Gamma-ray and neutrino signatures of unstable dark matter
}

\author{
David Tran ${ }^{* i}$ \\ Technical University of Munich \\ E-mail: david.tran@ph.tum.de
}

\begin{abstract}
We investigate indirect neutrino and gamma-ray signatures of dark matter in scenarios where the dark matter is weakly unstable, but decays with a cosmological lifetime. In the light of recently observed anomalous results in measurements of high-energy cosmic-ray electrons and positrons, we examine constraints imposed by the upcoming generation of neutrino observatories. We also discuss the large-scale gamma-ray anisotropies caused by the decay of dark matter particles in the Galactic halo and estimate their magnitude for the dark matter decay modes that can reproduce the anomalous cosmic-ray measurements.
\end{abstract}

Identification of Dark Matter 2010-IDM2010

July 26-30, 2010

Montpellier France

\footnotetext{
${ }^{*}$ Speaker.

${ }^{\dagger}$ In collaboration with L. Covi, M. Grefe, A. Ibarra and C. Weniger
} 


\section{Introduction}

While there is overwhelming evidence for the existence of copious amounts of non-luminous matter in the Universe, little is known about the physical nature of this dark matter. Among its unknown properties is the lifetime of the dark matter. While it is certainly plausible that the dark matter is perfectly stable, the only immediate lower bound on the dark matter lifetime is set by its presence in the Universe today, $\tau_{\mathrm{DM}} \gtrsim 10^{17} \mathrm{sec}$. If the dark matter is in fact unstable, it may be possible to detect its decay products as exotic contributions to the fluxes of photons, neutrinos or charged cosmic rays.

Indeed, over the last couple of years, cosmic-ray telescopes have made a number of unexpected observations that might indicate the presence of an exotic primary cosmic-ray component. Namely, the PAMELA telescope observed a steep rise in the positron fraction extending up to at least 100 $\mathrm{GeV}$ [1], in conflict with expectations from standard astrophysical processes. Furthermore, the Fermi Gamma-ray Space Telescope observed an $e^{+} e^{-}$flux [2] that is harder than expected, also pointing to the possible presence of additional primary component. At the same time, no hadronic excess of antiprotons was observed [3]. Many attempts have been made to interpret the anomalous behaviors of the leptonic cosmic rays in terms of dark matter, as well as more conventional astrophysical sources such as pulsars.

In many well-motivated models the dark matter particles decay with lifetimes that greatly exceed the age of the Universe. Thus, we entertain here the possibility that the dark matter is indeed composed of weakly unstable particles which decay mostly into charged leptons, thus constituting the source of the cosmic-ray anomalies while avoiding the overproduction of antiprotons. This interpretation suggests a scale for the dark matter mass of a few $\mathrm{TeV}$ and a lifetime of the order $10^{26}$ sec [4]. Although this lifetime exceeds the age of the Universe by several orders of magnitude, the resulting fluxes of charged cosmic rays, gamma rays and neutrinos can be in the observable range. In the following, we discuss two complementary methods of probing the region of the parameter space suggested by the cosmic-ray anomalies.

\section{Neutrino Signatures of Unstable Dark Matter}

Neutrinos constitute an important probe to constrain decaying dark matter, since their production is almost inevitable in scenarios involving charged leptons among the decay products of the dark matter. Namely, neutrinos can be produced directly in the decay of dark matter particles, e.g. $\psi_{\mathrm{DM}} \rightarrow \ell^{+} \ell^{-} v$ for fermionic dark matter. Alternatively, secondary neutrinos can be produced in the decay of charged leptons originating from dark matter, or when the dark matter decays into hadronic final states. Since they do not diffuse or lose energy, neutrinos can therefore provide some important and relatively direct spectral and directional information about the decay of dark matter into ordinary matter.

A major problem in the detection of a possible dark matter signal in neutrinos is the presence of large atmospheric backgrounds [5] which often mask the comparatively feeble signals from dark matter decay. The task is made easier, however, by the fact that for the relatively large dark matter masses under consideration here, the corresponding neutrino energies are in the range of hundreds of $\mathrm{GeV}$ to a few $\mathrm{TeV}$, where atmospheric backgrounds decrease to a point where the hypothetical 

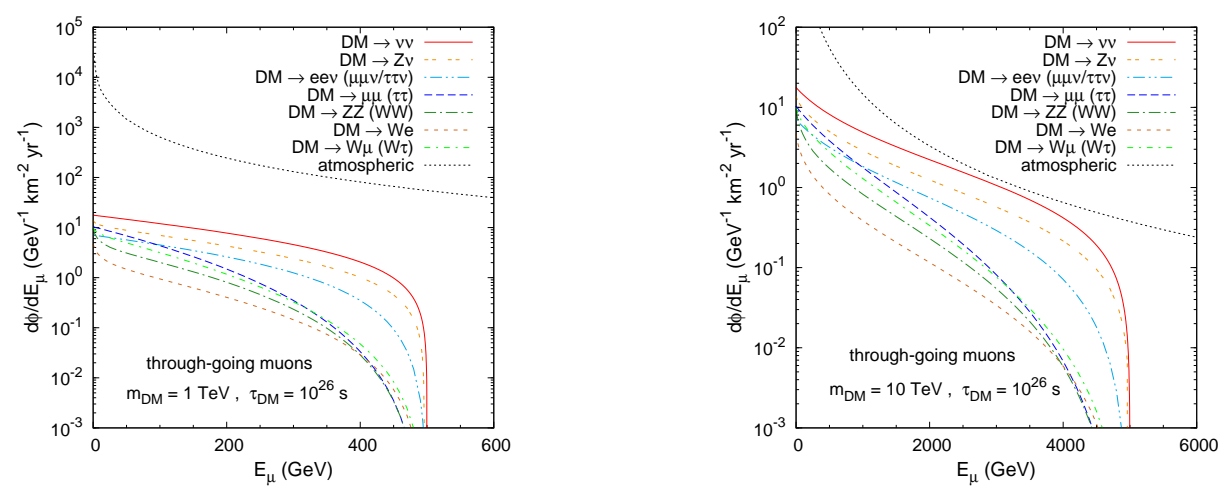

Figure 1: Flux of through-going muons in SuperKamiokande induced by neutrinos from dark matter decay. Left: Muon flux for various dark matter decay channels for a mass of dark matter mass of $1 \mathrm{TeV}$ and a lifetime of $10^{26} \mathrm{sec}$, as indicated by the cosmic-ray anomalies [9]. Right: Same, but for a mass of $10 \mathrm{TeV}$.
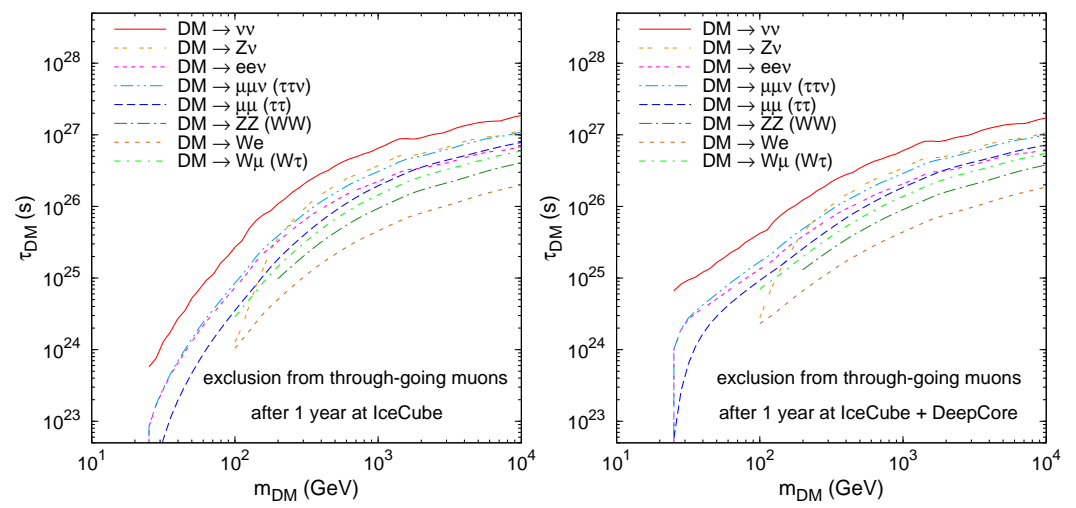

Figure 2: Projected IceCube exclusion limits from the non-observation of a signifcant excess of neutrinoinduced through-going muon events after one year of operation. Left: Exlusion limits for IceCube. Right: Exclusion imits for IceCube with the DeepCore subdetector.

dark matter signal becomes comparable to the backgrounds. Nevertheless, the detection of a small exotic contribution remains challenging. In the case of decaying dark matter, the best statistical significance of a dark matter-induced neutrino signal is achieved for full-sky observations, as opposed to observations focused on regions with a high dark matter density such as the center of the Galaxy. Due to the large propagation distances, neutrino flavor information is essentially erased, implying that the same signal is present in all flavors, independent of the original flavor composition. In the energy range of interest here, muon neutrinos from dark matter decay scatter deep-inelastically, triggering a hadronic shower and a muon track in the detector, which provides the clearest observational signature. In fig. 1, we show the flux of through-going muons at SuperKamiokande triggered by neutrinos from dark matter decay in various decay channels for sample dark matter masses of $1 \mathrm{TeV}$ and $10 \mathrm{TeV}$ to illustrate how the signals compare to the atmospheric background. The various neutrino spectra were obtained using PYTHIA 6.4 [6].

The non-observation of a neutrino-induced excess of muon events can be used to constrain the parameter space of dark matter mass vs. lifetime, which is essentially unconstrained by existing data. However, it turns out that the upcoming generation of neutrino telescopes of $\mathrm{km}^{3}$ dimensions 

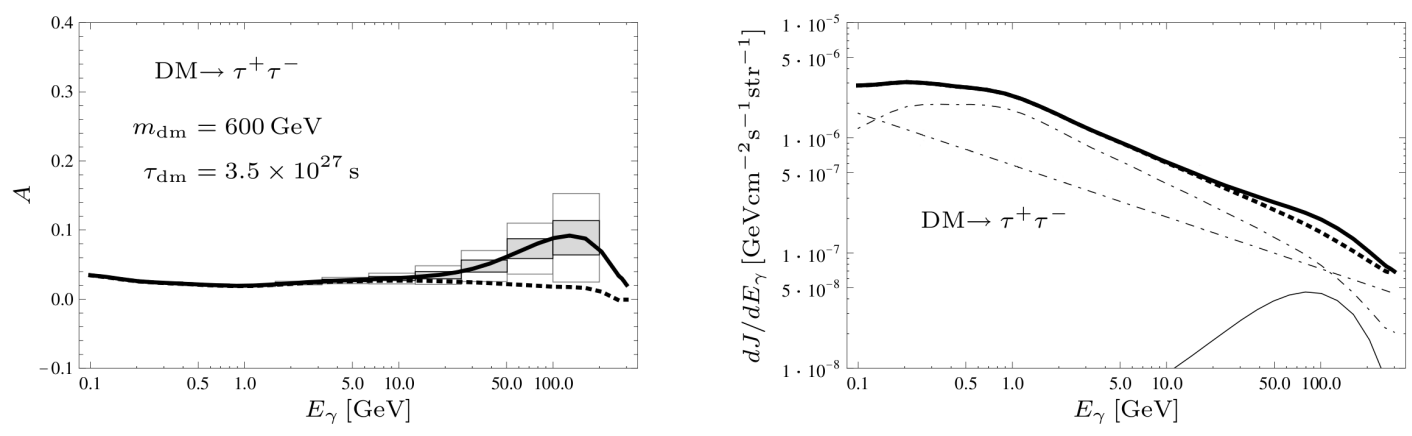

Figure 3: Left: Anisotropy in the total gamma-ray flux from the decay of dark matter into tau leptons, $\psi_{\mathrm{DM}} \rightarrow \tau^{+} \tau^{-}$, where the dark matter mass is $m_{\mathrm{DM}}=600 \mathrm{GeV}$ and the dark matter lifetime is $\tau_{\mathrm{DM}}=$ $3.5 \times 10^{27} \mathrm{sec}$. The dotted line shows the anisotropy of the Galactic foreground as predicted by GalProp, while the solid line shows the anisotropy of foreground + dark matter signal. The boxes indicate estimates of the statistical errors for one year and five years of Fermi LAT observations, respectively. Right: Total gamma-ray flux including Galactic foreground emission, extragalactic background and dark matter signal.

should be able to constrain the parameter space at a level relevant to the cosmic-ray anomalies within a few years of observations [7] (see also [8]). We show the projected limits we find for IceCube in various dark matter decay modes in fig. 2. We also illustrate the effects of adding the DeepCore subdetector, which can significantly increase the sensitivity at lower energies. If the dark matter interpretation of the cosmic-ray anomalies is correct, a corresponding signal in neutrinos should be detectable within the near future. Should a signal be observed, however, the discrimination between different dark matter decay channels seems almost impossible from neutrino observations, as different decay modes yield very similar signatures at neutrino observatories. To this end, complementary information from other channels like gamma-ray observations is needed.

\section{Large-Scale Gamma-Ray Anisotropies from Dark Matter Decay}

The decay of dark matter particles in the Galactic halo into gamma rays generally induces a dipole-like anisotropy in the gamma-ray flux due to the offset between our position and the center of the Galaxy [9]. This anisotropy, which contains contributions from both prompt photons from dark matter decay and inverse Compton scattering of electrons, is relatively mild on large angular scales compared to the case of dark matter annihilation, where the bulk of the emission is expected from the region close to the Galactic center. Nevertheless, the angular dependence of the signal is qualitatively different from the Galactic foreground and might therefore be used to identify a contribution from dark matter decay. While a detailed mapping of the angular profile of a possible gamma-ray signal from dark matter may be impossible to extract from observational data due to the presence of the Galactic foreground emission, an anisotropy might still be detected in the overall flux, i.e., in the sum of Galactic emission and dark matter signal, from large patches in opposite directions in the sky. Therefore, to quantify the overall anisotropy, it is convenient to define an anisotropy parameter $A$ as the normalized difference in the flux between the hemisphere in the 

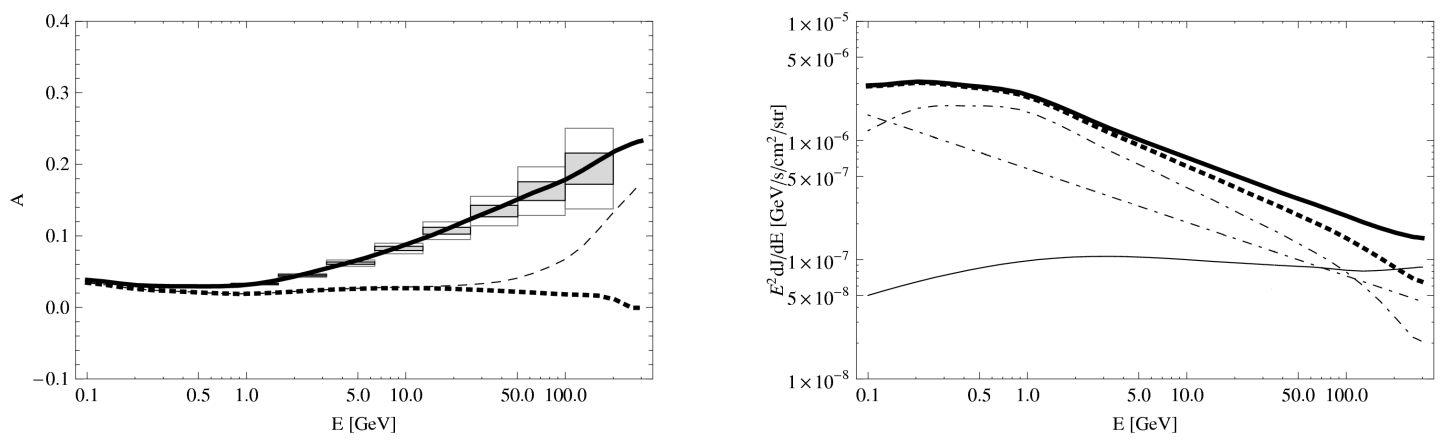

Figure 4: Same as fig. 3, but for the decay $\psi_{\mathrm{DM}} \rightarrow \mu^{+} \mu^{-} v$ with a dark matter mass $m_{\mathrm{DM}}=2.5 \mathrm{TeV}$ and a dark matter lifetime $\tau_{\mathrm{DM}}=2.3 \times 10^{26} \mathrm{sec}$. These parameters yield a good agreement with the electron/positron data measured by PAMELA and Fermi LAT [4]. The dashed line in the left panel indicates the anisotropy in the total flux when including the dark matter signal, but not the inverse Compton scattering of electrons and positrons from dark matter decay.

direction of the Galactic center and the hemisphere in the direction of the Galactic anticenter,

$$
A=\frac{\bar{J}_{\mathrm{GC}}-\bar{J}_{\mathrm{GAC}}}{\bar{J}_{\mathrm{GC}}+\bar{J}_{\mathrm{GAC}}}
$$

Here, $\bar{J}_{\mathrm{GC}}\left(\bar{J}_{\mathrm{GAC}}\right)$ denotes the flux from the Galactic center (anticenter), averaged over the respective hemispheres and integrated over the appropriate energy bins. We exclude regions at low Galactic latitudes, $|b|<10^{\circ}$, to minimize contamination from sources in the disk. We generally find that the anisotropy in the overall flux can be large enough to be within the reach of present observations. For instance, we show in fig. 3 the anisotropy in the overall flux for the decay of dark matter into $\tau^{+} \tau^{-}$pairs for a dark matter mass of $600 \mathrm{GeV}$ and a lifetime that is fixed by the requirement that the total gamma-ray flux do not exceed the isotropic extragalactic background as determined by the Fermi collaboration [10]. Again, the energy spectrum of photons was determined using PYTHIA [6]. The anisotropy from the Galactic foreground is calculated from Galactic emission in the conventional GalProp model (model 44_500180) [11]. Interestingly, the anisotropy induced by dark matter decay exhibits an energy-dependence that is absent in the Galactic foreground. Thus, observation of a spectral feature of this kind in the anisotropy parameter serve as a hint of a contribution from dark matter.

We can also use the large-scale anisotropies to test the dark matter interpretations of the cosmic-ray anomalies. We plot in fig. 4 the anisotropy for one of the decay modes that gives a good fit to the PAMELA and Fermi LAT $e^{+} e^{-}$data, namely the three-body decay $\psi_{\mathrm{DM}} \rightarrow \mu^{+} \mu^{-} \nu$. The mass and lifetime are fixed by requiring a good fit to the PAMELA and Fermi LAT data. In this case, the anisotropy at high energies $\left(E_{\gamma} \gtrsim 100 \mathrm{GeV}\right)$ can be as large as $20 \%$. Likewise, large anisotropies are found for all of the dark matter decay modes that reproduce the measurements of leptonic cosmic rays. Thus, at least for a priori models for Galactic gamma-ray emission, the dark matter interpretations of the cosmic-ray anomalies predict the existence of relatively large gammaray anisotropies on large scales. At least from the statistical point of view, these anisotropies should be observable, as indicated by our estimates of the statistical errors for one and five years of Fermi LAT observations. Systematic uncertainties, however, will make the determination of 
the anisotropy parameter more difficult in practice. Overall, we find that a contribution from dark matter to the diffuse gamma-ray flux could be observed as a spectral feature in the form of an anisotropy which increases with the energy. On the other hand, excluding dark matter interpretations of the cosmic-ray anomalies from a non-observation of large anisotropies is more difficult, and would require an improved understanding of the Galactic foreground emission.

We have also analyzed the angular power spectrum of gamma rays from dark matter decay. The power spectrum is sensitive to fluctuations in the dark matter density on smaller scales including subhalos and the large-scale distribution of dark matter in the nearby Universe. In the case of decaying dark matter, the latter contribution is the relevant one since the contribution from subhalos is neglibible. However, it turns out that the small-scale fluctuations are expected to be hidden under photon noise in the energy range of interest here. Therefore, they should not be accessible to present telescopes like Fermi LAT. Nevertheless, a (non)-observation of angular fluctuations could help to distinguish between decaying and annihilating dark matter, since for annihilating dark matter these fluctuations are typically larger and potentially observable [12].

\section{Acknowledgements}

I would like to thank Laura Covi, Michael Grefe, Alejandro Ibarra and Christoph Weniger for pleasant collaborations. Support from the DFG cluster of excellence "Origin and Structure of the Universe" and the DFG Graduiertenkolleg "Particle Physics at the Energy Frontier of New Phenomena" is acknowleged.

\section{References}

[1] O. Adriani et al. [PAMELA Collaboration], Nature 458, 607 (2009) [arXiv:0810.4995 [astro-ph]].

[2] A. A. Abdo et al. [The Fermi LAT Collaboration], Phys. Rev. Lett. 102, 181101 (2009) [arXiv:0905.0025 [astro-ph.HE]].

[3] O. Adriani et al., Phys. Rev. Lett. 102, 051101 (2009) [arXiv:0810.4994 [astro-ph]].

[4] A. Ibarra, D. Tran, C. Weniger, JCAP 1001, 009 (2010). [arXiv:0906.1571 [hep-ph]].

[5] M. Honda, T. Kajita, K. Kasahara, S. Midorikawa and T. Sanuki, Phys. Rev. D 75, 043006 (2007) [arXiv:astro-ph/0611418].

[6] T. Sjostrand, S. Mrenna and P. Z. Skands, JHEP 0605, 026 (2006) [arXiv:hep-ph/0603175].

[7] L. Covi, M. Grefe, A. Ibarra and D. Tran, JCAP 1004, 017 (2010) [arXiv:0912.3521 [hep-ph]].

[8] S. K. Mandal, M. R. Buckley, K. Freese, D. Spolyar and H. Murayama, Phys. Rev. D 81, 043508 (2010) [arXiv:0911.5188 [hep-ph]].

[9] A. Ibarra, D. Tran and C. Weniger, Phys. Rev. D 81, 023529 (2010) [arXiv:0909.3514 [hep-ph]].

[10] A. A. Abdo et al. [The Fermi-LAT collaboration], Phys. Rev. Lett. 104, 101101 (2010) [arXiv:1002.3603 [astro-ph.HE]].

[11] A. W. Strong, I. V. Moskalenko, O. Reimer, Astrophys. J. 613, 962-976 (2004). [astro-ph/0406254].

[12] B. S. Hensley, J. M. Siegal-Gaskins and V. Pavlidou, Astrophys. J. 723, 277 (2010) [arXiv:0912.1854 [astro-ph.CO]]. 